\title{
A New Synthetic Method for Controlled Polymerization Using a Microfluidic System
}

\author{
Tao Wu, Ying Mei, João T. Cabral, Chang Xu, and Kathryn L. Beers* \\ Polymers Division, National Institute of Standards and Technology, Gaithersburg, Maryland 20899
}

Received March 18, 2004; E-mail: kathryn.beers@nist.gov

Polymer materials applications increasingly show sensitivity to single-chain variables such as molecular mass and copolymer composition. ${ }^{1-3}$ While many parallel synthesis methods developed by the pharmaceutical and life science communities are being applied to polymer synthesis, ${ }^{4,5}$ there remains a need to construct polymeric material "libraries" that explore a wide range of polymer structures with accuracy, flexibility, and rapid, often precise, changes.

Recent development of various analytical methods, such as micrototal analytical systems ( $\mu$ TAS), ${ }^{6,7}$ have also increased the methodologies available for building polymer libraries. Particularly in screening processes, measurements require less and less material, causing much of the parallel synthesis products (typically carried out on 1-20-g scales) to go to waste. From both a scientific and an industrial perspective, it is desirable to develop a simple and economic combinatorial synthetic method to generate libraries with ranges of controlled polymer molecular mass and architecture, and copolymers with diversity in composition to generate detailed maps to probe structure-property relationships.

Here we demonstrate a method using microfluidic technology to create a micro-environment for continuous controlled radical polymerization, analogous to large-scale continuous reactors commonly used in industrial processes. Varying either the flow rate or the relative concentrations of reactants (i.e., stoichiometry) controls the molecular properties of the products. Molecular variables, here molecular mass, can be varied continuously. Well-defined materials with narrow molecular mass distributions produced inside the microfluidic reactor can be made available for processing, such as further mixing or coating on surfaces. In fact, the scale of this reactor is well matched to new high-throughput techniques that measure behavior of polymer films and solutions. ${ }^{8-10}$

To fabricate the microchannel reactor, we employed a rapid device prototyping technique based on contact photolithography of a thiolene prepolymer. ${ }^{11}$ Compared to poly(dimethylsiloxane) devices, ${ }^{12,13}$ this thiolene material has significantly improved solvent resistance, as shown by previous swelling experiments. ${ }^{11}$ Our experiments also demonstrate that the device shows low oxygen permeability and good pressure stability, which is critical to sustain radical polymerization conditions. The device consists of a glass/ resin/glass-layered structure, which embeds a single channel (500 $\mu \mathrm{m} \times 600 \mu \mathrm{m}$ ), with two channels and an active mixing chamber at the inlet and a single outlet (Figure 1). The mixing chamber contains a small stir bar driven with a magnetic stir plate. The dimensions of this device are larger than typical microfluidics devices to accommodate the increasing viscosities of the reaction solution as the monomer conversion and polymer molecular mass increase. Still, this technique is limited to low conversion bulk or solution polymerizations. However, we do not consider this a major limitation, since many specialty polymers are of low molecular mass and are often prepared in solution.

Atom transfer radical polymerization (ATRP) of 2-hydroxypropyl

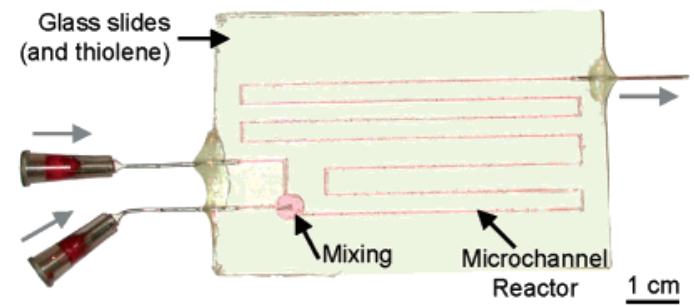

Figure 1. Photopolymerized microfluidic device fabricated with a rapid prototyping technique based on an optical thiolene resin. Gray arrows represent the direction of flow in the channels.

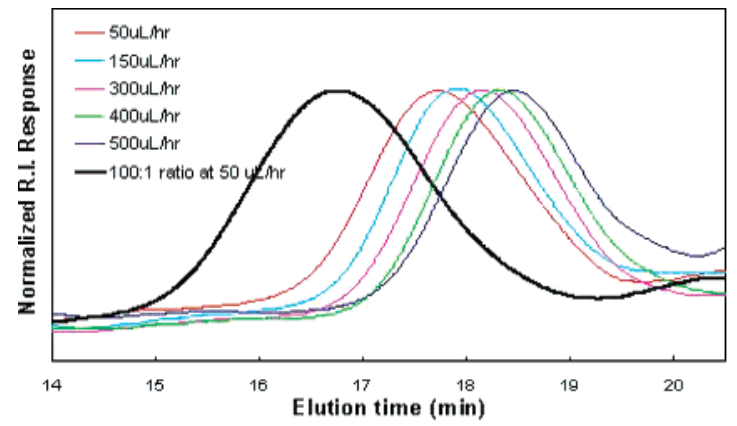

Figure 2. SEC trace of pHPMA produced from different pump rates with the ratio of initiator to monomer as 1:40 (thin lines) and 1:100 (bold line).

methacrylate (HPMA) was used to compare the kinetics and controllability in this reactor relative to batch reactions. ${ }^{14}$ Reagents were fed into the device via a dual rack syringe pump. Monomer and catalyst were added to one syringe, with a small amount of solvent (water/methanol, 50/50 by volume) to help the catalyst dissolve. The other syringe contained the same water/methanol mixture and initiator. The two syringes were set at identical pumping rates so that the final mixed solution had constant reagent concentrations. Two different initiator concentrations were tested ([initiator]: [monomer] of 1:100 and 1:40). A series of pump speeds were applied for each initiator concentration.

The microchannel reactor is designed to produce microgram samples-perhaps as trapped molecular gradients-for rapid on-line analysis, which our future work will explore. To validate our device through conventional analysis we required larger specimen volumes; thus, for each set of reaction conditions, we collected steady-state samples for extended times. Additional details can be found in the Supporting Information.

Size exclusion chromatography (SEC) data for representative samples of poly(2-hydroxypropyl methacrylate) products are shown in Figure 2. The series of thin lines represents the polymerization products with high initiator concentration (1:40), which were collected at varying flow rates. SEC analyses indicate symmetrical monomodal traces at all microchannel flow rates and show a polymer elution time shift as the pumping rate decreases and 


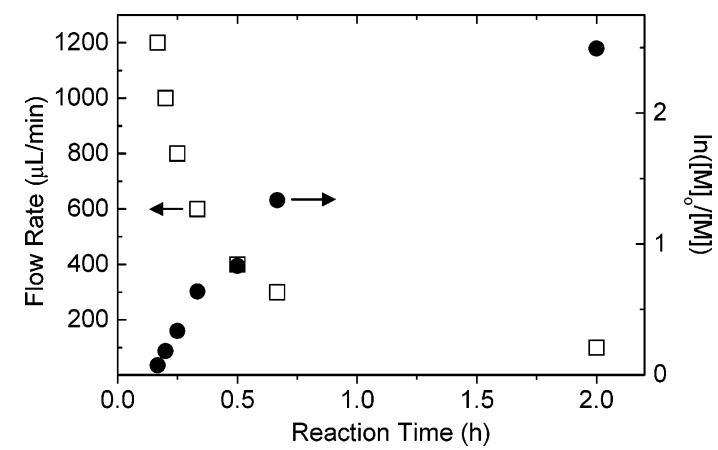

Figure 3. Kinetic data for ATRP of HPMA in a microchannel with initiator: HPMA of 1:40 resulting from different flow rates $(\square)$. Reaction time is shown as the independent variable for conventional representation of conversion data $(\bullet)[\mathrm{M}]_{0}=$ initial monomer concentration, $[\mathrm{M}]=$ monomer concentration during the reaction.

Table 1. Microchannel Polymerization of HPMA at Different Pumping Rates and Initiator Concentrations ${ }^{a}$

\begin{tabular}{|c|c|c|c|c|c|c|}
\hline [initiator]:[HPMA] & $\begin{array}{c}\text { pump rate } \\
(\mu \mathrm{L} / \mathrm{h})\end{array}$ & $\begin{array}{l}\text { rx time } \\
\text { (h) }\end{array}$ & conv. & $\begin{array}{c}M_{\mathrm{n}} \\
\text { (theory) }\end{array}$ & $\begin{array}{c}M_{\mathrm{n}} \\
(\mathrm{SEC})\end{array}$ & $M_{\mathrm{w}} / M_{\mathrm{n}}$ \\
\hline \multirow[t]{5}{*}{$1: 40$} & 50 & 2.00 & 0.92 & 5290 & 6240 & 1.21 \\
\hline & 150 & 0.67 & 0.74 & 4240 & 5560 & 1.19 \\
\hline & 300 & 0.33 & 0.47 & 3300 & 3950 & 1.26 \\
\hline & 400 & 0.25 & 0.29 & 2710 & 3300 & 1.27 \\
\hline & 500 & 0.20 & 0.17 & 1650 & 2770 & 1.32 \\
\hline $1: 100$ & 50 & 2.00 & 0.62 & 8990 & 12740 & 1.26 \\
\hline
\end{tabular}

${ }^{a}$ Standard uncertainties in conversion and SEC data are 5\% and $10 \%$, respectively. $M_{\mathrm{n}}=$ number average molar mass. $M_{\mathrm{w}} / M_{\mathrm{n}}=$ polydispersity.

reaction time increases. Polymer polydispersities remain low (1.191.32) and decrease with conversion as shown in Table 1 . The bold line in Figure 2 represents a higher-molecular mass polymer produced at a pumping rate of $100 \mu \mathrm{L} / \mathrm{h}$ with low initiator concentration (1:100).

Reaction time can be calculated on the basis of the residence time in the channel, which is manipulated with device design and flow control. To match residence time with the targeted reaction time, a channel volume of $200 \mu \mathrm{L}$ was chosen for HPMA. Because two syringe barrels are being discharged into the channel at the same speed, the solution flow rate is twice the pump speed. Figure 3 is a kinetic plot of $\ln \left([\mathrm{M}]_{0} /[\mathrm{M}]\right)$ vs time for the ATRP of HPMA inside a microchannel along with the relative flow rate corresponding to reaction time. The apparent rate of polymerization is constant up to high conversions with slight curvature thereafter, in good agreement with the batch reaction results reported by Save et al. ${ }^{11}$ At a flow rate of $100 \mu \mathrm{L} / \mathrm{h}$, the conversion rate reaches $92 \%$ with a number average molecular mass $\left(M_{\mathrm{n}}\right)$ of $6240 \mathrm{~g} / \mathrm{mol}$ and polydispersity $\left(M_{\mathrm{w}} / M_{\mathrm{n}}\right)$ of 1.21 .

Many research groups are developing on-chip chromatographic ${ }^{15}$ and spectroscopic methods ${ }^{16}$ that will be integrated with this approach in future work to enable microscale synthesis and characterization of well-defined polymeric materials in gradient libraries. We envision this technique will enable more comprehensive studies than are currently possible, spanning vast areas of molecular variable space. This will benefit areas of research using materials incorporating expensive, often bioderived monomers and macroinitiators. It is important to point out that by using additional inlets the component concentration and stoichiometry can be controlled by adjusting the relative pump speeds to obtain results similar to this work. Additional work relating to this feature is currently underway.

It is desirable to operate the devices at higher temperatures to prepare many of the more common polymers by controlled radical polymerization. Ongoing work is targeted at extending the longterm stability of the devices at temperatures up to $100{ }^{\circ} \mathrm{C}$. Several preliminary polymerizations were carried out at elevated temperatures, and the data suggest that well-defined materials are still produced under these conditions.

In conclusion, we successfully designed a microchannel reactor for controlled polymerization. The molecular mass of the polymer produced is governed by the flow rate or polymerization time. The monomer conversion agrees well with the bulk reaction kinetics reported in the literature. The reactor is convenient and inexpensive to build, with a versatile design that can be reconfigured and prototyped in less than a day. Our results suggest that this continuous microreactor can be used to produce libraries of materials for direct integration with high-throughput processing and characterization methods. For example, our future work includes expanding the range of materials prepared by this method and linking the output to mixing and measurement techniques to study the influence of molecular composition and architecture on phase behavior and mechanical properties of polymer materials.

Acknowledgment. We thank Drs. Eric Amis and Michael Fasolka for helpful discussions. Reserve funding from the director of the Materials Science and Engineering Laboratory at NIST is gratefully acknowledged. This work was carried out at the NIST Combinatorial Methods Center (NCMC; www.nist.gov/combi).

Supporting Information Available: Synthesis and methods. This material is available free of charge via the Internet at http://pubs.acs.org.

\section{References}

(1) Webster, O. W.; Science 1991, 251, 887-893.

(2) Thompson, L. F. Resist Processing. In Introduction to Microlithography, 2nd ed.; Thompson, L. F., Willson, C. G., Bowden, M. J., Eds.; American Chemical Society: Washington, DC, 1994; pp 269-274.

(3) Mansky, P.; Russell, T. P.; Hawker, C. J.; Pitsikalis, M.; Mays, J. Macromolecules 1997, 30, 6810-3813.

(4) Brocchini, S.; James, K.; Tangpasuthadol, V.; Kohn, J. J. Am. Chem. Soc. 1997, 119, 4553-4554.

(5) Meier, M. A. R.; Hoogenboom, R.; Schubert, U. S. Macromol. Rapid Commun. 2004, 25, 21-23.

(6) Manz, A.; Harrison, D. J.; Verpoorte, E.; Fettinger, J. C.; Ludi, H.; Widmer, H. M. Chimia 1991, 45, 103-105.

(7) Harrison, D. J.; Fluri, K.; Seiler, K.; Fan, Z.; Effenhauser, C. S.; Manz A. Science 1993, 261, 895-897.

(8) Harrison, C.; Stafford, C. M.; Beers, K. L.; Karim, A.; Amis, E. J.; VanLandingham, M. R.; Kim, H.-C.; Volken, W.; Miller, R. D.; Simonyi, E. E. Nat. Mater. 2004, in press.

(9) Smith, A. P.; Douglas, J. F.; Meredith, J. C.; Amis, E. J.; Karim, A. Phys. Rev. Lett. 2001, 87, 015503-1.

(10) Hudson, S. D.; Phelan, F. R.; Handler, M. D.; Cabral, J. C.; Migler, K. B.; Amis, E. J. Appl. Phys. Lett. 2004, in press.

(11) Harrison, C.; Cabral, J. T.; Stafford, C. M.; Karim, A.; Amis, E. J. J. Micromech. Microeng. 2004, 14, 153-158.

(12) Xia, Y. N.; Whitesides, G. M.; Angew. Chem., Int. Ed. 1998 37, 550575 .

(13) Quake, S. R.; Scherer, A. Science 2000, 290, 1536-1540.

(14) Save, M.; Weaver, J. V. M.; Armes, S. P.; McKenna, P. Macromolecules 2002, 35, 1152-1159.

(15) McEnery, M.; Tan, A. M.; Alderman, J.; Patterson, J.; O’Mathuna, S. C.; Glennon, J. D. Analyst 2000, 125, 25-27.

(16) Pan, T.; Kelly, R. T.; Asplund, M. C.; Woolley, A. T. J. Chromatogr., A 2004, 1027, 231-235.

JA048432N 